\title{
Linear concentrating collector as an air heater in the heating system of building in Polish climatic conditions
}

\author{
Magdalena Nemśs,a ${ }^{1,}$ Agnieszka Manikowska ${ }^{1}$ and Artur Nemś ${ }^{1}$ \\ ${ }^{1}$ Wroclaw University of Technology, Faculty of Mechanical and Power Engineering, Poland
}

\begin{abstract}
The article presents the analysis of the performance of a concentrating collector in the heating system of a residential building. Air was used as the working fluid. The heating requirements of the building were determined for each day of the year. The amount of direct irradiation reaching the absorber's surface on all the days of the year was determined with the use of hourly meteorological data for Wroclaw, shared by the Ministry of Infrastructure and Growth. It was assumed that the collector is equipped with a tracking system working in one axis. Calculations and comparisons were made for the amount of solar irradiation for three values of the receiver's inclination angle: $\beta_{1}=60^{\circ}, \beta_{2}=90^{\circ}$ and $\beta_{3}=30^{\circ}$. Statistical method was used in order to determine the optimum inclination of the mirror and the amount of flowing air. This method involves creating a plan of experiment with three levels of changeability for two input factors. In the last stage, the amount of heat obtained from the installation during all the days of the year was analysed. The gains were juxtaposed on the diagram with the building's heat demand. The analysis has shown that the heat requirements can be met only partially.
\end{abstract}

\section{Introduction}

The solar energy market in Poland has been dominated by flat solar collectors. Concentrating collectors are quite rare, and their manufacturers usually use water as the working medium. Selection of medium includes choosing appropriate construction parameters of the collector; the degree of concentration of mirror cannot be too high so that the final temperature of water obtained was not too high. Concentrators are used for purposes of heating of buildings, unlike countries with high insolation; in solar power plants, to run technological processes such as utilization, or production of process heat. The use of air as the working medium is not that popular. In literature on the subject there is research on flat solar collectors that heat the air. The main subject of this research is heat exchange intensification through changing the absorber's design. Its profile is changed: fins [1,2], wire mesh [3] or other elements such as internal beds made of scrap metal are added. However, this does not change the low temperature range on the basis of which flat collectors operate. There are few manuscripts and papers devoted to concentrating air collectors. Research conducted by scientists from Norway, who analysed different types of absorbers for solar concentrating parabolic dish [4], can be considered significant, whereas Turkish researchers studied the cone-shaped collector, which was ultimately used as a solar cooker [5].

Climatic conditions in Poland make solar collectors used in heating on a seasonal basis, mainly for generation of hot water in houses. It is associated with low insolation and winter temperatures which freeze the working medium. An important advantage of concentrating collectors is the possibility of obtaining high temperatures of the medium, even in the winter period. Further in this article the concept of a heating system in a singlefamily building based on a large-size, linearly focusing air collector is described.

\section{The heating system concept}

The main element of the analysed heating system is a focusing tube collector that heats the air. The hot working medium from the collector is distributed around a building through tubes (Fig. 1). As a backup system that supplements heat shortage a biomass fireplace that heats the air and uses the same heat distribution system around the house was used. The selection of the collector's working medium was not accidental. Despite a low thermal capacity, the air has many advantages. These include: universal accessibility, safety in case of installation leakage, and a simple structure, which translates into a low price of structural elements of the system. Another advantage is that the air from the collector can be directly used in the heat distribution installation in the building.

Calculations were made for the heating system dedicated for a single-family building with an area of $82 \mathrm{~m}^{2}$. As the geographic location the city of Wroclaw, located in the western part of Poland, was chosen.

\footnotetext{
a Corresponding author: magdalena.nems@pwr.edu.pl
} 
The heat demand for the house calculated according to the methodology of the Ministry was $45 \mathrm{kWh} / \mathrm{m}^{2}$ year.

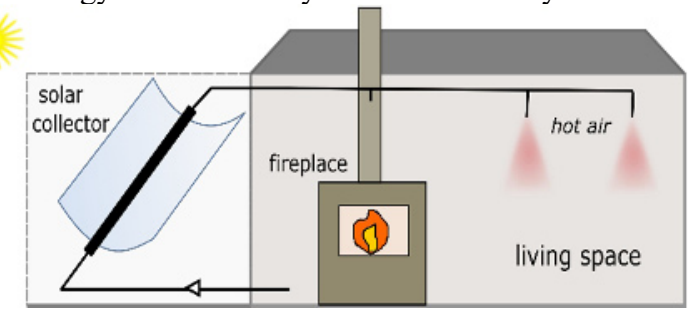

Figure 1. Schematic diagram of the analysed system.

To be able to conduct a detailed analysis of operation of the solar heating system the daily demand for heat in the building was calculated. For this purpose, a definition of heating degree-days was adopted [6]. The number of heating degree-days was calculated using the following equation (1) [6]:

$$
S d=\sum_{i=1}^{12} \sum_{i=1}^{31}\left(t_{i}-t_{e}(d)\right)
$$

where:

$t_{i}$ - base temperature, i.e. the adopted ambient temperature for which in the building there is thermal comfort, $t_{e}(d)$ - average daily temperature on a given day [7].

It was assumed that a given day is part of the heating period if the average daily temperature on a given day is lower than the base temperature. For the needs of calculations it was assumed that the base temperature for Wroclaw was $15^{\circ} \mathrm{C}$, and an additional relation was introduced:

$$
\left\{\begin{array}{c}
\text { if } t_{e}(d) \geq 15^{\circ} \mathrm{C} \text { then } S d(d)=0 \\
\text { if } t_{e}(d)<15^{\circ} \mathrm{C} \text { then } S d(d)=\left(t_{i}-t_{e}(d)\right)
\end{array}\right.
$$

On the basis of the relation (2) the daily number of degreedays was calculated for each day of the year for Wroclaw. This allowed calculating the daily demand for heat from the equation (3) [6]:

$$
Q_{h d}=Q_{h} \cdot \frac{\left(t_{i}-t_{e}(d)\right)}{s d}
$$

where:

$Q_{h}$ - annual heat demand.

The daily demand for heat is shown in Fig. 2.

Additionally, it was assumed that the heating system should be activated when for 3 days in a row the temperature is below $15^{\circ} \mathrm{C}$; in this case, the heating season lasts from mid-September to mid-May. In the remaining months the building does not have to be heated.

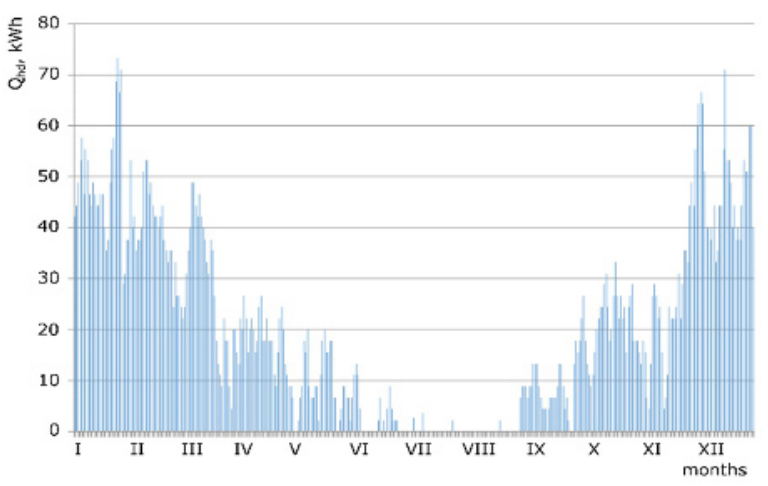

Figure 2. Daily heat demand.

\section{Collector as an air heater}

To examine the annual work of the collector and its efficiency its model was required. It was taken from [8]. The basic values used in the calculations are shown in Fig. 3. The copper tube in which there is the medium (air) was surrounded by a glass shield. In the space between the tube and the shield there was vacuum.

It was assumed that a 7-metre absorber has a selective coating - black chrome. The transparent shield is made of glass with a low content of $\mathrm{Fe}_{2} \mathrm{O}_{3}$ and with a thickness of $3 \mathrm{~mm}$. The adopted width of the collector's mirror was $3 \mathrm{~m}$, and the internal diameter of the absorber was $D W=0.096 \mathrm{~m}$, the outer diameter was $D_{z}=0.1 \mathrm{~m}$, and the diameter of the absorber's shield was $D_{c}=0.14 \mathrm{~m}$. The heat obtained from the collector was calculated on the basis of an equation by Hottel-Whillier-Bliss (4) [8]:

$$
Q_{u}^{*}=A_{f} F_{R}\left(S-\frac{A_{p}}{A_{f}} U_{g}\left(T_{w 1}-T_{a}\right)\right)
$$

where:

$A_{f}$ - non-shaded area of the aperture,

$F_{R}$ - heat removal coefficient from the receiver,

$S$ - solar radiation absorbed by the receiver,

$A_{p}$ - surface of the receiver,

$U_{g}$ - losses through transparent cover,

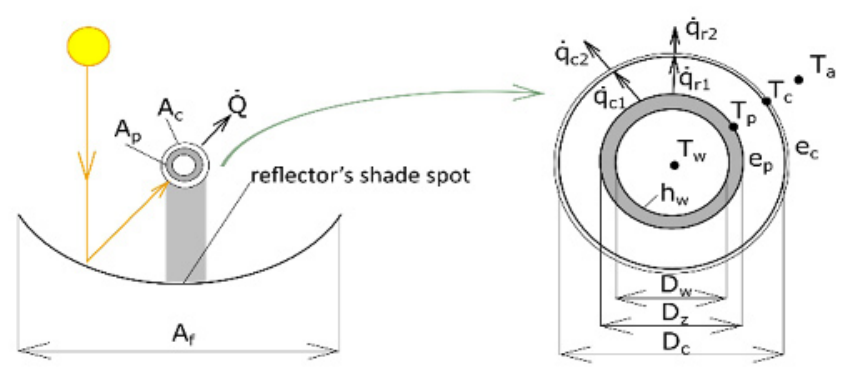

Figure 3. The symbols adopted in the analysis of the concentrating collector, where: $T$-temperature, $D$ - diameter, $q$-heat transfer flux, $e$ - emissivity, $h$ - heat transfer coefficient [based on 8]. 
$T_{w 1}$ - inlet temperature of the medium (it was assumed that the air is taken from the room and $T_{w l}=20^{\circ} \mathrm{C}$ was adopted),

$T_{a}$ - ambient temperature.

Collector efficiency expressed by equation 5 [8]:

$$
\eta_{m}=\frac{Q_{u}^{*}}{A_{\text {f gross }} \cdot G_{b}}
$$

where:

$A_{f \text { gross }}$ - total area of the aperture,

$G_{b}$ - density of direct radiation.

The formula for the efficiency of the model collector was determined using the iterative method. The formula included its size, solar radiation and power output.

\subsection{Analysis of solar radiation}

To a large extent the efficiency of a solar collector depends on solar radiation falling on the collector. In the case of concentrating collectors it is direct radiation. For this reason, the concentrating collector must have a follow-up system, which will direct the optical system throughout the day towards the sun. The analysed air heater had one axis of rotation. Additional three angles of inclination of the collector towards the horizontal plane were analysed: 30, 60 and 90 degrees.

The amount of radiation that reached the plane of the collector directly can be calculated using the following relation (6) [8].

$$
G_{b}=G_{o n} \cdot \cos \theta_{\beta} \frac{\mathrm{w}}{\mathrm{m}^{2}}
$$

where:

$G_{o n}$ - radiation flux density measured on a normal plane directed towards radiation,

$\theta_{\beta}$ - angle of incidence of radiation on the receiver's plane measured between the direction of direct radiation, and the normal plane directed towards the receiver.

To be able to make calculations of density of radiation a new angle of incidence of radiation on a plane of a moving concentrating collector was determined. For this purpose, relation (7) was used which allows calculating angle $\theta_{\beta}$ by taking into account the so-called optimum angle $\Psi$, for which the concentrating collector rotates optimally towards the current direction of sunlight [9].

$$
\begin{gathered}
\cos ^{2} \theta_{\beta}=\cos \psi[\sin \beta \sin \varphi \cos (\alpha-\gamma)+\cos \beta \cos \varphi]+ \\
+\sin \psi \sin \varphi \sin (\alpha-\gamma)
\end{gathered}
$$

where:

$\psi$ - optimum angle of rotation of the concentrating collector around its diagonal axis,

$\beta$ - inclination of the receiver towards the horizon (three variants of inclination of the collector were assumed: $\beta_{1}=30^{\circ}, \beta_{2}=60^{\circ}, \beta_{3}=90^{\circ}$ ),

$\varphi$ - zenith angle, the angle of incidence of solar radiation on a horizontal plane, $\alpha$ - solar azimuth,

$\gamma$ - receiver's azimuth, deviation from the local meridian measured relative to the southern direction; $\gamma=0$ was adopted.

The optimum angle of rotation of the collector around the diagonal axis is calculated from equation (8) [9]. It falls within the range of $-90^{\circ}<\psi<+90^{\circ}$.

$$
\tan \psi_{\text {opt }}=\frac{\sin \varphi \sin (\alpha-\gamma)}{\sin \beta \sin \varphi \cos (\alpha-\gamma)+\cos \beta \cos \varphi}
$$

On the basis of relation (6) - (8) the amount of direct radiation that reaches the collector at any hour of the day throughout the year was calculated for the three variants of inclination. The maximum values of radiation received for angle $30^{\circ}$ amounted to $550 \mathrm{~W} / \mathrm{m}^{2}$, for $60^{\circ}-490 \mathrm{~W} / \mathrm{m}^{2}$, and for $90^{\circ}-320 \mathrm{~W} / \mathrm{m}^{2}$. The obtained amounts were compared throughout the year and for further analysis the angle of inclination of $\beta=30^{\circ}$ was assumed (Fig. 4).

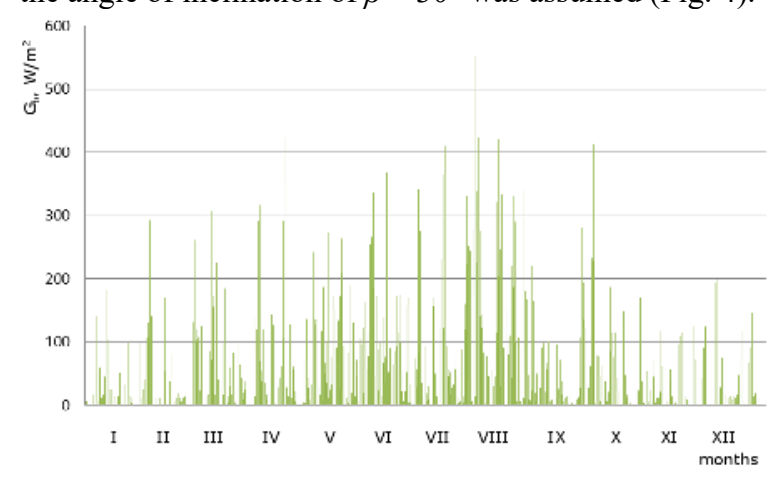

Figure 4. Amount of direct radiation that reaches the collector for the angle of inclination of $\beta=30^{\circ}$.

\subsection{The experiment planning method}

A key issue before conducting the analysis of annual work of the conceptual system was to determine a dependence for the operating efficiency of the collector.

The analysis of the collector's work shows that variable parameters that affect the efficiency of the system include the density of direct radiation and the value of a given air flow stream. When determining the effect of more than one factor on the result of a given process, special plans are used to simplify the courses of research and development of results. To determine the equation, the procedure of conduct of a static experiment planning was used for two input factors at three levels of variation, in short PS/DK $3^{2}[10]$. The use of the PS/DK $3^{\text {n }}$ plan guarantees accurate analysis of the process, even if it had been unknown, and allows obtaining a mathematical model of an examined issue in the form of a second degree polynomial [10].

In the selected programme the input factors take on value on three levels of variation [10]:

- higher, marked as +1 .

- medium, also called basic or zero, marked as 0 ,

- lower, marked as -1 . 
The equation that is obtained in the three-level planning has the form of a second degree polynomial in the form of equation (9) [10]:

$$
y=b_{0}+\sum b_{k} x_{k}+\sum b_{k k} x_{k}^{2}+\sum b_{k j} x_{k} x_{j}
$$

where:

$x_{k}, x_{j}, \ldots$ - input factors,

$b_{0}, b_{k}, \ldots$ - regression coefficients,

$y$ - result factor.

For the tested model the three-level plan was with two input factors - direct radiation and flow stream - was adopted. Levels of variation, which are shown in Table 1, were selected for the given factors. The values were selected on the basis of collected meteorological data and predicted possibilities of changes in the flowing air stream.

Table 1. Parameters of input factors.

\begin{tabular}{|c|l|c|c|}
\hline Item & \multicolumn{1}{|c|}{ Parameter } & $G_{b}\left(\mathrm{~W} / \mathrm{m}^{2}\right)$ & $m^{*}(\mathrm{~kg} / \mathrm{s})$ \\
\hline 1 & Zero level (0) & 450 & 0.03 \\
\hline 2 & Upper level (+) & 800 & 0.05 \\
\hline 3 & Lower level (-) & 100 & 0.01 \\
\hline 4 & $\begin{array}{l}\text { Range } \\
\text { of changes }\end{array}$ & 350 & 0.02 \\
\hline 5 & $\begin{array}{l}\text { Codes } \\
\text { of variables }\end{array}$ & $x_{1}=\left(\frac{G_{b}-450}{350}\right)$ & $x_{2}=\left(\frac{m^{*}-0.03}{0.02}\right)$ \\
\hline 6 & Result factor & \multicolumn{2}{|c|}{$y=\eta_{s y s}$} \\
\hline
\end{tabular}

The efficiency of the collector was calculated from equation (9) after calculating regression coefficients [10], and pre-coded factors (Table 2).

Table 2. Values of regression coefficients.

\begin{tabular}{|c|c|}
\hline$b_{0}$ & 0.5956 \\
\hline$b_{11}$ & -0.0057 \\
\hline$b_{22}$ & -0.0129 \\
\hline$b_{12}$ & 0.0002 \\
\hline$b_{1}$ & 0.0074 \\
\hline$b_{2}$ & 0.0198 \\
\hline
\end{tabular}

Table 3 shows a list of input factors and efficiency values calculated for these factors from model $\eta_{m}$ of the collector, and efficiency values calculated from experiment planning $\eta_{\text {sys }}$. The obtained results were similar. The efficiency calculated using the experiment method has almost the same values as the one calculated from the model. Differences are observed only in hundredths of a percent therefore the calculations made were considered correct.

On the basis of the above calculations a universal formula for efficiency of the modelled collector, dependent on direct radiation and the air flow stream in the absorber, was obtained. This formula has the following form (10):

$$
\begin{gathered}
\eta=0.5956+0.0074 \cdot \frac{G_{b}-450}{350}+ \\
+0.0198 \cdot \frac{m^{*}-0.03}{0.02}-0.0057 \cdot\left(\frac{G_{b}-450}{350}\right)^{2}+
\end{gathered}
$$

$$
\begin{array}{r}
-0.0129 \cdot\left(\frac{m^{*}-0.03}{0.02}\right)^{2}+ \\
+0.0002 \cdot \frac{G_{b}-450}{350} \cdot \frac{m^{*}-0.03}{0.02}
\end{array}
$$

Table 3. A comparison of the efficiency of the collector from model $\eta_{m}$ and the efficiency calculated from experiment

\begin{tabular}{|c|c|c|c|c|}
\hline \multicolumn{5}{|c|}{ planning $\eta_{\text {sys. }}$} \\
\hline Item & $G_{b}, \mathrm{~W} / \mathrm{m}^{2}$ & $m^{*}, \mathrm{~kg} / \mathrm{s}$ & $\eta_{m}, \%$ & $\eta_{s y s}, \%$ \\
\hline 1 & 800 & 0.05 & 60.42 & 60.43 \\
\hline 2 & 800 & 0.03 & 59.73 & 59.72 \\
\hline 3 & 800 & 0.01 & 56.43 & 56.43 \\
\hline 4 & 450 & 0.05 & 60.25 & 60.24 \\
\hline 5 & 450 & 0.03 & 59.57 & 59.56 \\
\hline 6 & 450 & 0.01 & 56.27 & 56.29 \\
\hline 7 & 100 & 0.05 & 58.91 & 58.91 \\
\hline 8 & 100 & 0.03 & 58.24 & 58.25 \\
\hline 9 & 100 & 0.01 & 55.02 & 55.01 \\
\hline
\end{tabular}

The obtained equation was substituted to the calculated data. The changing efficiency of the collector for radiation $G_{b} \quad\left(\beta_{1}\right)$ at different values of $m^{*}$ was analysed. Three values of the flow stream were compared: $m_{l}=0.05$ $\mathrm{kg} / \mathrm{s}, m_{2}=0.03 \mathrm{~kg} / \mathrm{s}$, and $m_{3}=0.01 \mathrm{~kg} / \mathrm{s}$. The results were presented in the form of a graph shown in Fig. 5. Fig. 6 shows the changing efficiency of the collector with three different values of solar radiation: $G_{b l}=100 \mathrm{~W} / \mathrm{m}^{2}$, $G_{b 2}=450 \mathrm{~W} / \mathrm{m}^{2}, G_{b 3}=800 \mathrm{~W} / \mathrm{m}^{2}$.

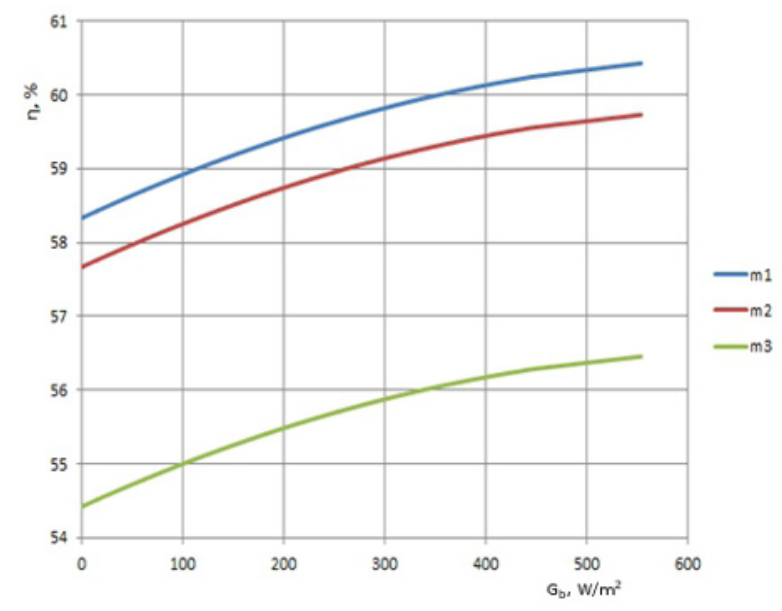

Figure 5. Collector efficiency change chart depending on available solar radiation at different values of the flow stream.

Charts presented in Fig. 5 and 6 show that the efficiency of the collector is highest for flow stream $m_{l}=0.05 \mathrm{~kg} / \mathrm{s}$, and solar radiation $G_{b 3}=800 \mathrm{~W} / \mathrm{m}^{2}$. 


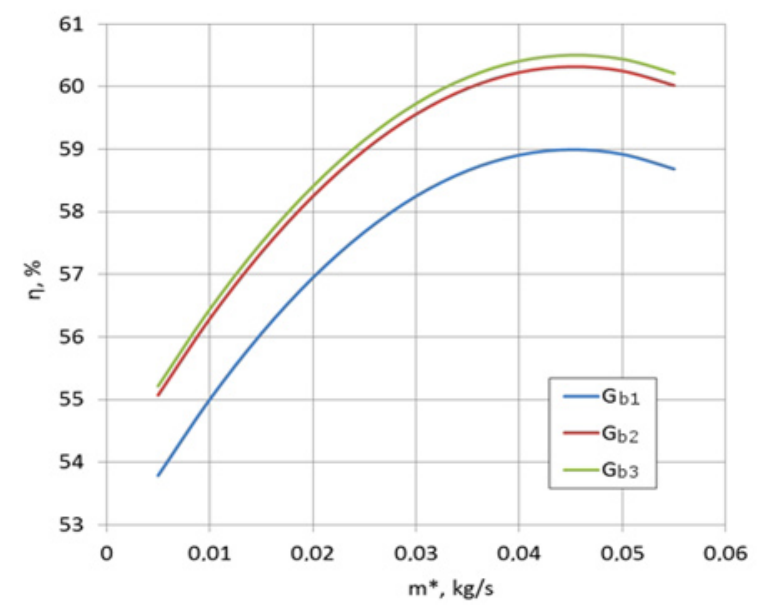

Figure 6. Collector efficiency change chart depending on the flow stream at different values of solar radiation $G$.

\section{Analysis of annual operation of the system}

After determining the general formula for the efficiency of the concentrating collector depending on the flow stream of the medium and the amount of solar radiation, it was possible to determine the changeability of the amount of power output of the collector per year. Using formula (5) the thermal power of the analysed collector was calculated for various combinations of inclinations of the device and the flow stream of the medium.

After making the calculations it can be seen that the greatest power output of the analysed concentrating collector is observed for angle $\beta=30^{\circ}$ and flow stream $m=0.05 \mathrm{~kg} / \mathrm{s}$. The maximum power output of the collector was $Q=168 \mathrm{kWh}$ at the end of July. During the heating season the heating power reaches $90 \mathrm{kWh}$. For the variant with angle $\beta=60^{\circ}$ the maximum thermal power of the collector does not exceed $160 \mathrm{kWh}$, and for variant $\beta=90^{\circ}-100 \mathrm{kWh}$. In the case of the analysed variants, thermal power of the collector changes by a few kWh only.

On the basis of Figure 7, the degree to which the solar collector meets the heat demand of the building can be assessed. The highest heat demand of a singlefamily house are in the months from September to May.
Whereas the collector has the most power in the summer months from May to September, when the greatest amount of sunlight is available. In the months from March to October the designed solar collector is enough to heat the building however in the remaining months of the heating season and additional heating source is required. In the months from November to February heat gains due to the short day are insufficient. Due to the fact that the working medium of the concentrating collector is air and it is used to heat the building, a fireplace with a hot air distribution function was selected as the additional heating source.

\section{Summary and conclusions}

The most popular methods of heating in Poland are traditional solutions such as gas furnaces, stoves, solid fuel furnaces or urban heating. Heat distribution in rooms is carried out with the use of the water system. In recent times, it has been recognized that air heating is increasingly used for heating houses. Hot air distribution fireplaces, but also air solar collectors, are used for this purpose. The latter are dominated by flat collectors. Concentrating collectors are not that common, especially in Poland.

This manuscript analysed the work of a concentrating solar collector. Heat exchange calculations were made to determine efficiency, and the amount of solar radiation reaching the plane of the collector was determined. Thanks to the experiment planning method the general formula for the efficiency of the collector, which depends on the flow stream of the working medium and the amount of solar radiation reaching the collector, was worked out. The changing efficiency of the collector for three different variants of inclination and three variants of flow of the medium was analysed. It has been shown that the best solution, in which the efficiency of the system is the highest, is the variant with the angle of inclination of $\beta=30^{\circ}$ and the flow stream of $m=0.05 \mathrm{~kg} / \mathrm{s}$. For this solution the collector reaches the greatest thermal power. The maximum amount of energy exceeds 160 $\mathrm{kWh}$, whereas in the least efficient variant $\left(\beta=90^{\circ}\right.$ and $m=0.05 \mathrm{~kg} / \mathrm{s}$ ) the amount of energy gained from the collector is less than $100 \mathrm{kWh}$.

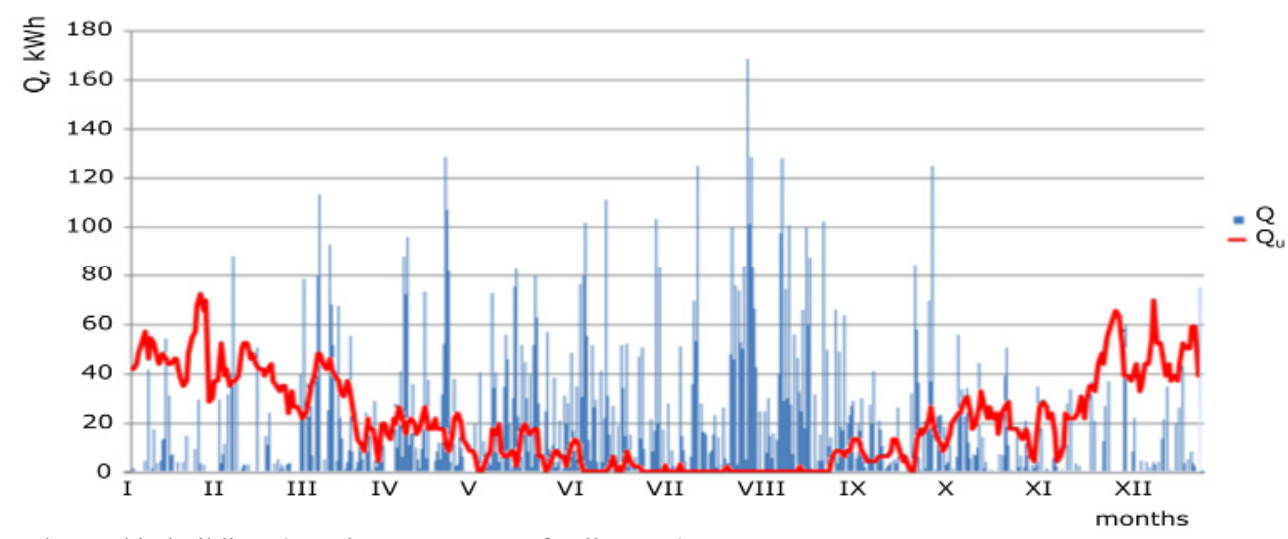

Figure 7. Heat demand in building $Q_{u}$ and power output of collector $Q$. 
The last step in the analysis was to compare heat demand in the building with the amount of heat provided by the collector for the best of the considered variants. The designed collector covers the thermal needs of the building completely in the months from March to October. In the remaining months the heat from the collector is not enough to heat the entire building therefore an auxiliary heating source, which is a biomass furnace, was chosen. In both cases heat is delivered to the heated rooms through insulated ducts. Both systems can constitute an integral whole. Then, the building will be provided thermal comfort in every day of the year, and renewable energy sources will be used to heat it. It is also possible to use a heat accumulator which could accumulate excess heat and supplement heat deficiency when the temperature in the building drops below thermal comfort.

\section{References}

1. J. Muñoz, A. Abánades, Appl. Energy 88, (2011)

2. J. Kasperski, M. Nemś, Appl. Therm. Eng. 58, (2013)

3. P. Verma, L. Varshney, Sustain. Energy Technol. Assess., 10 (2015)

4. H.B. Madessa, T. Veslum, J. Løvseth, O.J. Nydal, 30th ISES Biennial Solar World Congress 2011, SWC 20113, (2011)

5. I.T. Togrul, D. Pehlivan, Appl. Therm. Eng. 25, (2005)

6. J. Dopke, The calculation of monthly number of heating degree (in Polish): www.ogrzewnictwo.pl, accessed : 22.04.2016

7. Meteorological data for Wroclaw: http://air.wroclaw.pios.gov.pl/, accessed : 22.04.2016

8. Z. Pluta, Theoretical basis of photothermal conversion of solar energy (in Polish), (Publishing house of Warsaw University of Technology, Warsaw, 2006)

9. J. Kasperski, M. Lewkowicz, S. Pietrowicz, (in Polish), District Heating, Heating, Ventilation 14, 6/7 (2007)

10. M. Korzyński, Methodology of the experiment (in Polish), (Scientific-Technical Publisher, Warsaw, 2006) 